\begin{tabular}{|c|c|}
\hline Title & Room-Temperature Protonation-Driven On-Demand Metal-Insul ator Conversion of a Transition Metal Oxide \\
\hline Author(s) & Katase, Takayoshi; Endo, Kenii; Tohei, Tetsuya; Ikuhara, Y uichi; Ohta, Hiromichi \\
\hline Citation & $\begin{array}{l}\text { A dvanced Electronic Materials, 1(7), } 1500063 \\
\text { https://doi.org/10.1002/ael m.20150063 }\end{array}$ \\
\hline Issue Date & 2015-07 \\
\hline Doc URL & http:/hdl.handle.net/2115/62320 \\
\hline Rights & $\begin{array}{l}\text { (1) 2015 Wiley-VCH, This is the accepted version of the foll lowing article: A dvanced Electronic Materials } V \text { ol.1, } \\
\text { 1500063, which has been published in final form at } \\
\text { http://onlinelibrary.wiley .com/doi } 10.1002 / \text { aelm.201500063/abstract. }\end{array}$ \\
\hline Type & article (author version) \\
\hline File Information & Katase_aelm.1500063.pdf \\
\hline
\end{tabular}

Instructions for use 
This is the pre-peer reviewed version of the following article:

T. Katase, K. Endo, T. Tohei, Y. Ikuhara, and H. Ohta, Advanced Electronic Materials 1, 1500063 (2015)., which has been published in final form at http://onlinelibrary.wiley.com/woll/doi/10.1002/aelm.201500063/abstract.

\section{Room-temperature-protonation-driven on-demand metal-insulator conversion of a transition metal oxide}

Takayoshi Katase ${ }^{1, *}$, Kenji Endo ${ }^{1}$, Tetsuya Tohei ${ }^{2}$, Yuichi Ikuhara ${ }^{2}$, and Hiromichi Ohta ${ }^{1, *}$

${ }^{1}$ Research Institute for Electronic Science, Hokkaido University, N20W10, Sapporo 001-0020, Japan

${ }^{2}$ Institute of Engineering Innovation, The University of Tokyo, 2-11-16 Yayoi, Bunkyo-ku, Tokyo 113-8656, Japan

*E-mail: katase@es.hokudai.ac.jp, hiromichi.ohta@es.hokudai.ac.jp

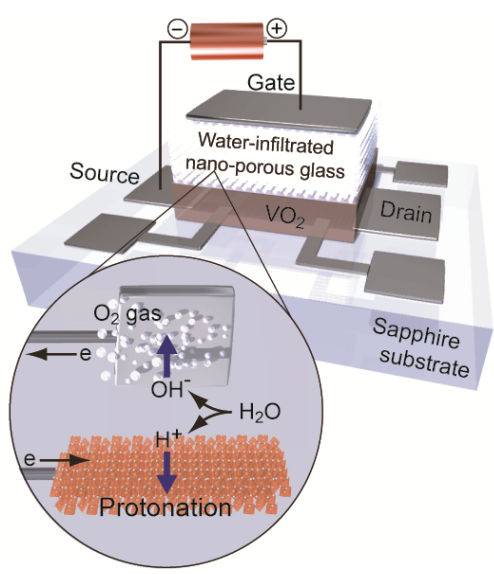

Room-temperature protonation and metal-insulator conversion of a transition metal oxide (TMO) was demonstrated by all-solid-state thin-film transistor with vanadium dioxide using a water-infiltrated nanoporous glass as a solid electrolyte. This promising result can provide a novel route for TMO-based solid-state electro-optical devices, in particular, smart windows for on-demand infrared shielding.

Keywords: protonation, solid state electrochemistry, transition metal oxide, thin film transistor 
Hydrogen in transition metal oxides (TMOs) commonly exist as protons $\left(\mathrm{H}^{+}\right)$through covalent $\mathrm{O}-\mathrm{H}$ bonding with the surrounding oxide ions $\left(\mathrm{O}^{2-}\right)^{[1]}$ and act as shallow donors that donate an electron to TM cations, ${ }^{[2]}$ resulting in a dramatic change in the electro-optical properties. ${ }^{[3]}$ Although $\mathrm{H}^{+}$has historically been viewed as the most ubiquitous impurity, ${ }^{[4]}$ recent studies suggest that it may reveal the potential characteristics in TMOs. Protonation of TMOs is the most ideal technique to modulate their functions, even as compared with state-of-the-art modulation techniques, ${ }^{[5,6]}$ due to their intrinsic nonvolatile operation, ${ }^{[7,8]}$ but protonation is not typically utilized for solid-state devices because of imperative high-temperature annealing treatment in hydrogen source. Although one solution for room temperature (RT) protonation of TMOs is liquid-phase electrochemistry through electrolysis of an aqueous solution, which has been a facile method to protonate TMOs at $\mathrm{RT},{ }^{[9,10]}$ it is unsuited for practical purposes due to liquid-leakage problem. Hence, protonation/deprotonation in solid-state devices has yet to be realized in practical applications.

Herein, we focus on vanadium dioxide $\left(\mathrm{VO}_{2}\right)$ to demonstrate solid-state protonation and conversion of the electronic states from an insulator to a metal at $\mathrm{RT} \mathrm{VO}_{2}$ is classically well-known thermochromic TMO, which shows metal-insulator (MI) transition at the transition temperature $\left(T_{\mathrm{MI}}\right)$ of $68{ }^{\circ} \mathrm{C} .{ }^{[11]}$ The MI transition accompanies a structural change from a low-temperature monoclinic to a high-temperature tetragonal (rutile-type) structure, which causes reconstruction of electronic structures to open up a charge gap of $\sim 0.6 \mathrm{eV},^{[12]}$ resulting in the abrupt change of electrical and optical properties at the MI transition. In particular, the dramatic change of optical transmittance in infrared region while maintaining visible transparency has potential for heat-blocking smart window, which enables us to intelligently regulate and utilize sunlight, leading to energy saving in architectures. These features of $\mathrm{VO}_{2}$ offer several advantages compared to visible-color switching of conventional 
electrochromic devices. However, the relatively high $T_{\mathrm{MI}}$ of $\mathrm{VO}_{2}$ makes it awkward to use at appropriate temperatures for practical applications.

Protonation of $\mathrm{VO}_{2}\left(\mathrm{H}_{x} \mathrm{VO}_{2}\right)$ is one of the effective ways to reduce $T_{\mathrm{MI}}$ and modulate the electronic state from an insulator to a metal via hydrogen spillover through thermal annealing of the $\mathrm{VO}_{2}$ nanobeam in $\mathrm{H}_{2}$ / vacuum conditions. ${ }^{[13]}$ MI phase modulation has also been achieved by electrostatic-charge doping with ionic-liquid-gated transistors ${ }^{[14,15]}$ and epitaxial strains in thin films, ${ }^{[16]}$ but protonation of $\mathrm{VO}_{2}$ via a chemical route is the most ideal to engineer the MI transition characteristics due to the intrinsic non-volatile operations similar to a conventional electrochromic device based on $\mathrm{H}_{x} \mathrm{WO}_{3} \cdot{ }^{[3]}$ However, protonation of $\mathrm{VO}_{2}$ requires a high-temperature heat treatment, ${ }^{[17-19]}$ and the uptake of $\mathrm{H}^{+}$into $\mathrm{VO}_{2}$ was extremely slow at RT, i.e. the chemical solubility of $\mathrm{H}_{x} \mathrm{VO}_{2}$ was very small at $x=0.08 .{ }^{[17]}$ Controlling the MI phase transition by RT protonation has technological importance as a step toward practical uses.

Here we propose a new solid-state device for on-demand-protonation of $\mathrm{VO}_{2}$ thin films at RT using a thin film transistor (TFT) combined with water-electrolysis. ${ }^{[20]}$ Figure 1a schematically illustrates the device structure of a water-electrolysis TFT composed of water-infiltrated nanoporous glass as a gate insulator, which serves as solid electrolyte. ${ }^{[20]}$ The gate insulator consists of an amorphous $12 \mathrm{CaO} \cdot 7 \mathrm{Al}_{2} \mathrm{O}_{3}(\mathrm{a}-\mathrm{C} 12 \mathrm{~A} 7)$ thin film with nanoporous structure (Hereafter this film is abbreviated as CAN). C12A7 is hydroscopic material, and water vapor in air is automatically absorbed into the CAN film due to the capillary effect of the interconnected nanopores. ${ }^{[20]}$ A gate bias application induces water electrolysis in the CAN gate insulator, and produced electro-active $\mathrm{H}^{+}\left(\mathrm{H}_{3} \mathrm{O}^{+}\right)$and $\mathrm{OH}^{-}$ions can be used to protonate and deprotonate the $\mathrm{VO}_{2}$ channel layer, respectively. The water-electrolysis TFT can be considered as a nanosized electrochemical cell that enables reversible 
protonation/deprotonation in a high-electric field. Consequently, the $\sim 10$-nm-thick $\mathrm{VO}_{2}$ layer was reversibly altered from an insulator to a metal by changing the external gate voltage up to $35 \mathrm{~V}$ at RT. The protonation was clearly accompanied by the structural change from monoclinic $\mathrm{VO}_{2}$ to tetragonal $\mathrm{H}_{x} \mathrm{VO}_{2}$ phase. The $\mathrm{MI}$ phase modulation in protonated $\mathrm{VO}_{2}$ under an applied voltage at RT offer a new route to all-solid-state smart windows for on-demand infrared shielding, and the present results lead to a universal approach to modulate the electrical and optical properties of TMOs via water-electrolysis-induced protonation.

The TFT structure was fabricated on 20-nm-thick $\mathrm{VO}_{2}$ epitaxial films (channel size: 800- $\mu \mathrm{m}$ long and $400-\mu \mathrm{m}$ wide) grown on (11 $\overline{2} 0)$ sapphire substrates by pulsed laser deposition. Crystallographic characterization of the $\mathrm{VO}_{2}$ epitaxial film is summarized in Figure $\mathrm{S} 1$ in Supporting Information. $T_{\mathrm{MI}}$ of as-grown $\mathrm{VO}_{2}$ films was $65{ }^{\circ} \mathrm{C}$, which is similar to $68{ }^{\circ} \mathrm{C}$ of bulk $\mathrm{VO}_{2} \cdot{ }^{[11]}$ Figure $1 \mathrm{~b}$ shows an optical micrograph of the $\mathrm{VO}_{2}$-based water-electrolysis TFT. Metallic Ti films were used for Source (S), Drain (D) and Gate (G) electrodes. A 200-nm thick CAN film was used for a gate insulator as solid electrolyte, where the film was deposited at RT under an oxygen pressure of $5 \mathrm{~Pa}$ to realize a nanoporous structure. (The relative density of the CAN film was $71 \%$ with respect to $2.92 \mathrm{~g} \mathrm{~cm}^{-3}$ of a dense C12A7 film). ${ }^{[20]}$ Figure 1c shows Z-contrast, high-angle, annular dark-field scanning transmission electron microscope (HAADF-STEM) image of the cross-sectional TFT structure. The numerous dark spots with 10-20 nm diameters indicate that the high-density nanopores were incorporated in the $\mathrm{CAN}$ film. For the protonation of $\mathrm{VO}_{2}$ by water-electrolysis TFTs, we focused on the electronic structure of parent (insulating) $\mathrm{VO}_{2}$. The energy-band diagram of $\mathrm{VO}_{2}{ }^{[21]}$ with respect to the electric potentials of oxidation and reduction of $\mathrm{H}_{2} \mathrm{O}$ (Figure $\mathrm{S} 2$ in Supporting Information) shows that the conduction band minimum is below the electric potential of cathode reaction $\left(2 \mathrm{H}^{+}+2 \mathrm{e}^{-} \rightarrow \mathrm{H}_{2}\right)$ and the valence band maximum is above that of anode reaction $\left(\mathrm{H}_{2} \mathrm{O} \rightarrow 1 / 2 \mathrm{O}_{2}+2 \mathrm{H}^{+}+2 \mathrm{e}^{-}\right)$. Thus, the proton 
can be expected to move into and get out from $\mathrm{VO}_{2}$ channel layer without the gas generation by applying positive and negative electric-fields, respectively.

$\mathrm{VO}_{2}$-based water-electrolysis TFTs were characterized at RT by measuring sheet resistance $\left(R_{\mathrm{S}}\right)$ and thermopower $(S)$ after applying and subsequently switching off gate bias. It should be noted that $S$-values can be used to easily evaluate the metal-insulator conversion because they basically reflect the energy differential of density of state at Fermi energy and are sensitive to significant changes in the electronic structure of $\mathrm{VO}_{2}$ at $T_{\mathrm{MI} \cdot}{ }^{[22]}$ Figures $2 \mathrm{a}$ and b plot the gate voltage $\left(V_{\mathrm{g}}\right)$ dependence of $R_{\mathrm{S}}$ and $S$-values at RT for $\mathrm{VO}_{2}$-based water-electrolysis TFTs, respectively. The dependence was measured immediately after the bias application for 10 minutes at each positive voltage from $+5 \mathrm{~V}$ to $+35 \mathrm{~V}$. A double-digit decrease in $R_{\mathrm{s}}$ from the virgin state $\left(68 \mathrm{k} \Omega \mathrm{sq}^{-1}\right)$ to $0.5 \mathrm{k} \Omega \mathrm{sq}^{-1}$ at $+35 \mathrm{~V}$ was clearly observed, and the $R_{\mathrm{s}}$ continued to decrease by positive $V_{\mathrm{g}}$ application. Meanwhile, $|S|$-value drastically decreased form $200 \mu \mathrm{V} \mathrm{K} \mathrm{K}^{-1}$ of insulating parent $\mathrm{VO}_{2}$ phase ${ }^{[22]}$ as the positive $V_{\mathrm{g}}$ increased up to $+30 \mathrm{~V}$ and became saturated at $43 \mu \mathrm{V} \mathrm{K}{ }^{-1}$, which is close to that of metallic $\mathrm{H}_{x} \mathrm{VO}_{2}$ bulk $\left(\sim 35 \mu \mathrm{V} \mathrm{K}^{-1}\right){ }^{[19]}$ These results are discussed by comparing the electronic structures of insulating $\mathrm{H}_{x} \mathrm{VO}_{2}\left(T<T_{\mathrm{MI}}\right)$ and metallic $\mathrm{H}_{x} \mathrm{VO}_{2}\left(T>T_{\mathrm{MI}}\right)$. Schematic electronic structures around Fermi energy are shown in Figure S3 in Supporting Information. $\mathrm{H}^{+}$doping into insulating $\mathrm{H}_{x} \mathrm{VO}_{2}$ provides electrons to the conduction band, and the gradient of DOS becomes moderate, resulting in the consequent reduction of $|S|$-values. On the other hand, $|S|$-values of metallic $\mathrm{H}_{x} \mathrm{VO}_{2}$ are constant at $\sim 35 \mu \mathrm{VK}^{-1}$ at $T>T_{\mathrm{MI}}$. Therefore, the decrease in $|S|$-values and their saturation as $V_{\mathrm{g}}$ increases reflect a change in the electronic state from an insulator to a metal. The inset in Figure 2a summarizes the $V_{\mathrm{g}}$ dependence of $I_{\mathrm{g}}$ during the bias application at each $V_{\mathrm{g}}$. Although $I_{\mathrm{g}}$ at $V_{\mathrm{g}}=+5 \mathrm{~V}$ was small bellow $10 \mathrm{pA}, I_{\mathrm{g}}$ exponentially increased with respect to $V_{\mathrm{g}}$ from $+10 \mathrm{~V}$ to $+35 \mathrm{~V}$, suggesting that $I_{\mathrm{g}}$ originates from the ion current of water-electrolysis in the CAN film and electrochemical reaction of $\mathrm{VO}_{2}$ film. 
These findings suggest the device operation mechanism as follows. First, $V_{\mathrm{g}}$ application at $+5 \mathrm{~V}$ accumulates charge carriers at the $\mathrm{VO}_{2}$ film surface via a pure electrostatic field effect due to the negligible leakage current, resulting in the formation of parallel-plate capacitor (i.e. the electric field starts to be well applied on the surface of $\mathrm{VO}_{2}$ channel). Using a capacitance $(C)$ of $160 \mathrm{pF}$ for the CAN gate insulator, ${ }^{[20]}$ the accumulated sheet carrier density is estimated to be $1.6 \times 10^{12} \mathrm{~cm}^{-2}$ at $V_{\mathrm{g}}=+5 \mathrm{~V}$. A further gate bias application generates dissociated $\mathrm{H}^{+}$and $\mathrm{OH}^{-}$ions in the solid gate insulator, which are attracted to the surface of $\mathrm{VO}_{2}$ and $\mathrm{Ti}$, respectively. $\mathrm{H}^{+}$ions penetrate into the $\mathrm{VO}_{2}$ crystal $\left(\mathrm{H}_{x} \mathrm{VO}_{2}\right)$, while $\mathrm{OH}^{-}$ions form an oxidized $\mathrm{TiO}_{2}$ passivation layer on the Ti film surface or become $\mathrm{O}_{2}$ gas at the interface, where the electrostatic potential with linear distribution should be formed across CAN layer. Therefore, threshold voltage of the device operation for the water-electrolysis TFTs can be considered to be much higher than the $\mathrm{H}_{2} \mathrm{O}$ dissociation potential of $1.23 \mathrm{~V}$. The high electric field formed in the nanosized electrochemical cell should achieve RT protonation of the $\mathrm{VO}_{2}$ thin film, as illustrated in Figure $1 \mathrm{a}$. It should be noted that the protonated $\mathrm{VO}_{2}$ film is stable under ambient and vacuum conditions at RT. The retention time dependence of $R_{\mathrm{S}}$ monitored after switching the gate bias $(+20 \mathrm{~V})$ off is shown in Figure S4a in Supporting Information. Although slight decrease of $R_{\mathrm{s}}$ was observed in air, $R_{\mathrm{s}}$ are almost persistent following the removal of $V_{\mathrm{g}}$, confirming the non-volatility of device operation due to the chemical reaction.

Then, we performed reversible operation of $\mathrm{VO}_{2}$-based water-electrolysis TFTs, i.e. protonation and deprotonation by changing positive and negative gate bias. Figure $2 \mathrm{c}$ shows the temperature dependences of $R_{\mathrm{s}}$ before and after applying $V_{\mathrm{g}}$ alternately at $+20 \mathrm{~V}$ and -20 $\mathrm{V}$ at RT, where $R_{\mathrm{s}}-T$ was measured after switching off $V_{\mathrm{g}}$. The inset plots logarithm of $R_{\mathrm{s}}$ versus $T$ to show the MI transition clearly. $R_{\mathrm{s}}-T$ were reversibly modulated and recovered to 
initial state, where the ON/OFF ratio was $\sim 30$ at RT. However, unlike the previous report on bulk $\mathrm{H}_{x} \mathrm{VO}_{2},{ }^{[13]} T_{\mathrm{MI}}$ did not shift to lower temperature and was almost unchanged.

Considering that the $R_{\mathrm{s}}$ continues to decrease as $V_{\mathrm{g}}$ increases and the $|S|$-values reaches at that of metal $\mathrm{H}_{x} \mathrm{VO}_{2}$ at $V_{\mathrm{g}}=+30 \mathrm{~V}$ (Figures $2 \mathrm{a}$ and b), only the film surface region becomes metal due to an insufficient $\mathrm{H}^{+}$migration from the surface into bulk region. To estimate the thickness $(d)$ of metal $\mathrm{H}_{x} \mathrm{VO}_{2}$ layer, we used a simple bi-layer model of thermopower, ${ }^{[23]}$ where the parallel circuit composed of metal (M) and insulator (I) layers was considered to calculate the combined electromotive force. (The electromotive force depends on both the conductivity and thickness of each layer.) The observed $|S|$-value $\left(|S|_{\text {obsd. }}=43 \mu \mathrm{V}\right.$ $\left.\mathrm{K}^{-1}\right)$ of the present TFTs can be expressed by the equation of $|S|_{\mathrm{obsd}}=\left(\sigma_{\mathrm{sM}} \cdot|S|_{\mathrm{M}}+\sigma_{\mathrm{s}} \cdot|S|_{\mathrm{I}}\right) /\left(\sigma_{\mathrm{sM}}\right.$ $+\sigma_{\mathrm{sI}}$, where sheet conductance $\sigma_{\mathrm{sM}}$ and $\sigma_{\mathrm{sI}}$ are defined as $\sigma_{\mathrm{M}} \times d$ and $\sigma_{\mathrm{I}} \times(20-d)$, respectively. The physical properties of conductivity and $|S|$-values for the $\mathrm{M}$ and I phases are used from those of reported bulk $\mathrm{H}_{x} \mathrm{VO}_{2}{ }^{[19]}$ and virgin $\mathrm{VO}_{2}$ TFTs (Figures 2a and b), respectively. As a result, maximum thickness of metallic layer, after the $V_{\mathrm{g}}$ application at +35 $\mathrm{V}$, is estimated to be $11 \mathrm{~nm}$, confirming that the $\mathrm{VO}_{2}$ film surface was changed from an insulator to a metal state.

The MI transition of $\mathrm{VO}_{2}$ is strongly coupled with crystal structure change from an insulating monoclinic phase to a metallic tetragonal phase, which is accompanied by the disappearance of $\mathrm{V}^{4+}$-ion dimer. ${ }^{[12]}$ To validate the crystal structure change of protonated $\mathrm{VO}_{2}$ film, structural analysis was performed by electron diffraction with TEM at RT. Figures 3a and $\mathrm{b}$ present the nano-beam electron diffraction (ED) patterns observed at about $5 \mathrm{~nm}$ in depth from surface region of $\mathrm{VO}_{2}$ film, before (a) and after protonation (b) with an incident electron beam along the [010] direction of $\mathrm{VO}_{2}$. The initial ED pattern (a) is consistent with the monoclinic structure, where the diffraction spots of superstructures, indicated by arrows, 
originate from the formation of $\mathrm{V}^{4+}$-ion dimer. Following the protonation (b), the diffraction spots due to the superstructures disappear, demonstrating a transformation from the monoclinic to the tetragonal phase (Figures $3 \mathrm{c}$ and $\mathrm{d}$ ). This result indicates that crystal structure was changed by water-electrolysis-induced protonation of $\mathrm{VO}_{2}$, which provides evidence of metal-insulator conversion. It should be noted that, for heavily-protonated $\mathrm{H}_{x} \mathrm{VO}_{2}$ nanobeams ${ }^{[24]}$ and ionic-liquid-gated $\mathrm{VO}_{2} \mathrm{TFTs}^{\left[{ }^{[25]}\right.}$ their metallic phases were recently found to have the partially retained V3d orbital ordering. However, the pairing of $\mathrm{V}$ ions was not confirmed in the present ED patterns. Additional investigation is necessary to verify this inconsistency in the future.

The metal-insulator conversion must be reversibly controlled in a practical time for the application to electronic devices. Figures $4 \mathrm{a}$ and $4 \mathrm{~b}$ present $I_{\mathrm{g}}$ under $V_{\mathrm{g}}$ application and $R_{\mathrm{S}}$ at the OFF state versus number of gate pulses of $+20 \mathrm{~V}$ and $-20 \mathrm{~V}$ at RT, where each pulse duration is $1 \mathrm{~min}$. Upon the application of $V_{\mathrm{g}}$ within $1 \mathrm{~min}$, a large $I_{\mathrm{g}}$ of $\sim \pm 160 \mu \mathrm{A}$ was observed and consequently reversible switching of $R_{\mathrm{S}}$ was realized. The voltage switching of metal-insulator conversion demonstrates great potentials especially-for on-demand-operating smart windows advanced electronic devices based on protonated $\mathrm{VO}_{2}$. Here, we like to discuss a typical time scale of the switching process and its $V_{\mathrm{g}}$ dependence of $\mathrm{VO}_{2}$-based water-electrolysis TFTs. The switching speed of metal-insulator conversion can be considered to depend on water-electrolysis, ion migration in the solid electrolyte, and proton diffusion into $\mathrm{VO}_{2}$ film under $V_{\mathrm{g}}$ application. In particular, the proton diffusion should limit the operation speed in this case because of the low diffusion constant of proton at RT. Therefore, the device-operation speed should strongly depend on the density of protons accumulated at the surface of $\mathrm{VO}_{2}$ channel. Therefore, the speed of metal-insulator phase switching should basically depend on $V_{\mathrm{g}}$, because the density of protons ( $\equiv I_{\mathrm{g}}$ ) exponentially increases with $V_{\mathrm{g}}$. 
There has been a considerable debate concerning the field-induced MI phase conversion of ionic-liquid-gated $\mathrm{VO}_{2}$ TFTs, in which it results from a collapse of the Mott insulator due to electrostatically accumulated electrons at the film surface ${ }^{[14]}$ or arises from the formation of oxygen vacancies due to an electric-field-induced oxygen migration during the gating process. ${ }^{[15]}$ The latter one might be valid for CAN-gated water-electrolysis TFTs, but, in this case, the DC of $I_{\mathrm{g}}$ keeps unchanged, during $V_{\mathrm{g}}$ application, at extremely large value of $0.1-1 \mathrm{~mA}$ without the decay for $60 \mathrm{sec}$ at least (Fig. 4(a)) and it is hard to consider the CAN forms the capacitor structure. The present device seems to operate like rechargeable $\mathrm{Ni}-\mathrm{H}_{2}$ battery with constant ionic current, where linear electric-field induces the penetration of ions into each electrode. Therefore, electric-field effect on $\mathrm{VO}_{2}$ film surface should not be so high to induce oxygen vacancies in the film because of the lack of electric double layer at the interface. In addition, it is known fact that $\mathrm{H}^{+}$can penetrate into $\mathrm{VO}_{2}$ crystal and $\mathrm{H}^{+}$with smallest ionic radii (i.e. just proton without electron shell) ${ }^{[26]}$ is easily considered to penetrate into $\mathrm{VO}_{2}$ crystal, compared to the large cations of ionic liquids $\left(\sim 10^{2} \mathrm{pm}\right)$ such as HMIM-TFSI $^{[15]}$ (in this case, the oxygen vacancy mechanism might work.) Therefore, protonation should tend to take place more than electric-field-induced oxygen migration in the present device.

To investigate the pure electric-field effect on this $\mathrm{VO}_{2}$-TFT, we fabricated TFT structure with water-free gate insulator of a fully dense a-C12A7 film (i.e. the relative density of a-C12A7 film is $100 \%\left(2.9 \mathrm{gcm}^{-3}\right)$ and there is no water-containing nanopores in the film). ${ }^{[27]}$ The TFT characteristics at RT with the results of CAN-gated $\mathrm{VO}_{2}$ TFT are summarized in Supporting Information Fig. S5. The $I_{\mathrm{g}}$ for dry a-C12A7-gated $\mathrm{VO}_{2}$ TFT was $<1 \mathrm{nA}$ at most, which is $\sim 10^{6}$ times smaller than that of CAN-gated $\mathrm{VO}_{2}$ TFT, indicating that pure electric field effect is dominant. Although the $C$ of $210 \mathrm{pF}$ for a-C12A7 film is higher than that (160 pF) of CAN film, ${ }^{[20]}$ both the $R_{\mathrm{S}}$ and $S$-values have kept almost unchanged even at $V_{\mathrm{g}}=+40 \mathrm{~V}$ (The maximum $R_{\mathrm{s}}$ modulation ratio was $\sim 0.1 \%$ ). The MI phase conversion by 
electric-field effect was not observed in dry a-C12A7-gated $\mathrm{VO}_{2}$ TFTs and should originate from water-electrolysis in the CAN gate insulator.

To further confirm that the metallization of $\mathrm{VO}_{2}$ based water-electrolysis TFTs originates from protonation rather than the formation of oxygen vacancies, ${ }^{[15]}$ the protonated TFT was heated in a vacuum. Annealing temperature dependence of $R_{\mathrm{S}}$ for the protonated TFTs, measured at RT, is shown in Figure S4b in Supporting Information. $R_{\mathrm{s}}$ was nearly constant at annealing temperatures below $100{ }^{\circ} \mathrm{C}$, but significantly increased at $150{ }^{\circ} \mathrm{C}$ and $R_{\mathrm{S}}$ returned to the insulating state. These observations indicate that thermal deprotonation of $\mathrm{H}_{x} \mathrm{VO}_{2}$ occurred at $150{ }^{\circ} \mathrm{C}$, which is consistent with the result for bulk $\mathrm{H}_{x} \mathrm{VO}_{2}\left(87{ }^{\circ} \mathrm{C}^{[18]}\right)$. The increase of $R_{\mathrm{S}}$ in the TFTs upon heating in a vacuum supports that the device operation does not originate from the formation of oxygen vacancies.

Additionally, protonation of $\mathrm{VO}_{2}$ TFTs is further supported by the change of ED patterns on electron beam irradiation (Figure S6 in Supporting Information for ED patterns of the protonated $\mathrm{VO}_{2}$ TFTs as a function of irradiation time of electron beam). The ED patterns of protonated $\mathrm{VO}_{2}$ films changed and the diffraction spots of superstructures appeared as the irradiation time $(t)$ increased, where the ED pattern of $\mathrm{VO}_{2}$ TFTs at $t=90 \mathrm{sec}$ can correspond to M2-type monoclinic structure. ${ }^{[28]}$ These observations indicate that the tetragonal structure (metal) changed to monoclinic structure (insulator) upon electron beam irradiation at RT, which can be explained if thermal deprotonation occurs in a vacuum. A similar result was observed for p-type GaN:Mg thin films grown by MOCVD ${ }^{[29,30]}$; the obstacle hydrogen produced by $\mathrm{NH}_{3}$ dissociation can be removed from $\mathrm{GaN}: \mathrm{Mg}$ film by electron beam irradiation in a vacuum and accordingly p-type conduction was realized. Although it is difficult to completely rule out the contribution of oxygen vacancies,,these results should support the water-electrolysis-induced protonation of $\mathrm{VO}_{2}$ film at RT.

In summary, we demonstrated a new solid-state device for water-electrolysis-induced 
protonation and nonvolatile and reversible switching of metal-insulator conversion of $\mathrm{VO}_{2}$ at RT. We fabricated a transistor structure on an insulating $\mathrm{VO}_{2}$ thin film using water-infiltrated nanoporous glass (CAN) as a solid gate insulator, which acts as a solid electrolyte. A gate voltage application induced water electrolysis in the CAN gate insulator with large ion current i.e., nanosized electrochemical cell, and the produced protons were effectively incorporated and removed from $\mathrm{VO}_{2}$ film, which results in a highly reversible alternation of the electronic state from an insulator to a metal. The reversible protonation clearly accompanied a change in the $\mathrm{VO}_{2}$ crystal structure from insulating monoclinic to metal tetragonal phase. These findings provide a new pathway to realize all-solid-state electrochromic devices based on $\mathrm{VO}_{2}$ for energy-saving applications that reversibly change the infrared transmission, while maintaining the visible-light transparency. At this stage, the modulation thickness is limited to be $\sim 10 \mathrm{~nm}$ because the electric field is terminated at a thin layer of $\mathrm{VO}_{2}$ channel and protons cannot penetrate deeper into insulating $\mathrm{VO}_{2}$ layer. Indeed, it is difficult to apply this TFT structure to optical devices because, for practical use, a large change in transmittance is required and thus thicker film should be used. We consider that the all-solid state electrochromic device can be practical by changing the device structure to sandwich-type construction (e.g. ITO top electrode / CAN gate insulator $/ \mathrm{VO}_{2}$ film / $\mathrm{SnO}_{2}: \mathrm{F}$ bottom electrode) on glass substrate, where the electric field can be applied over the thickness of $\mathrm{VO}_{2}$ layer and the optical properties is largely modulated by RT protonation. The present approach should lead to the development of electro-optically active solid-state devices with TMO materials by engineering RT-protonation.

\section{Experimental section}

Device Fabrication: TFT structures (Figure1a) were fabricated on $20 \mathrm{~nm}$-thick $\mathrm{VO}_{2}$ epitaxial films using metal shadow masks. The dimensions of the channel were $400 \mu \mathrm{m}$ in width and $800 \mu \mathrm{m}$ in length. The $\mathrm{VO}_{2}$ channel layer was grown at $500{ }^{\circ} \mathrm{C}$ under $\mathrm{O}_{2}$ pressure $\left(P_{\mathrm{O} 2}\right)$ of 2.0 
Pa on $(11 \overline{2} 0) \quad \alpha-\mathrm{Al}_{2} \mathrm{O}_{3}$ substrates $\left(10 \times 10 \times 0.5 \mathrm{~mm}^{3}\right)$ by pulsed laser deposition $(\mathrm{PLD})$. A $\mathrm{KrF}$ excimer laser (wavelength of $248 \mathrm{~nm}$ ) was used to ablate a $\mathrm{V}_{2} \mathrm{O}_{5}$ polycrystalline target disk, where the laser energy fluence and the repetition rate were $3 \mathrm{~J} \mathrm{~cm}^{-2}$ pulse ${ }^{-1}$ and $10 \mathrm{~Hz}$, respectively. Afterwards the films were cooled to RT under the same oxygen pressure. The $\mathrm{VO}_{2}$ film with a monoclinic structure was heteroepitaxially grown on $\alpha-\mathrm{Al}_{2} \mathrm{O}_{3}$ substrate with an epitaxial relationship of (100)[010] $\mathrm{VO}_{2} \|(11 \overline{2} 0)[0001] \alpha-\mathrm{Al}_{2} \mathrm{O}_{3}$, which was confirmed by high-resolution XRD at RT (Figure S1 in Supporting Information). Second, 20-nm-thick metallic Ti films, which served as both the source and drain electrodes, were deposited by e-beam (EB) evaporation in a vacuum $\left(\sim 10^{-4} \mathrm{~Pa}\right.$, no substrate heating). Then, 200-nm-thick CAN gate insulator was deposited by PLD ( $\mathrm{KrF}$ excimer laser with energy fluence of $3 \mathrm{~J} \mathrm{~cm}^{-2}$ pulse $^{-1}$ ) at RT under $P_{\mathrm{O} 2}$ of $5 \mathrm{~Pa}$ to make CAN film nanoporous structure. ${ }^{[20]}$ The bulk density of the resultant CAN film was $2.07 \mathrm{~g} \mathrm{~cm}^{-3}$, evaluated by grazing incidence X-ray reflectivity, which corresponds to $71 \%$ of fully dense amorphous $\mathrm{C} 12 \mathrm{~A} 7$ glass $\left(2.92 \mathrm{~g} \mathrm{~cm}^{-3}\right){ }^{[20]} \mathrm{AC}$ conductivity of the CAN film was $3.7 \times 10^{-8} \mathrm{~S} \mathrm{~cm}^{-1}$ at RT, which was slightly smaller than $5.6 \times 10^{-8} \mathrm{~S} \mathrm{~cm}^{-1}$ of ultrapure water. Finally, a 50-nm-thick metallic Ti film, used as the gate electrode, was deposited by EB evaporation at RT.

Electrical transport measurement: Gate current $\left(I_{\mathrm{g}}\right)$ was measured as a function of gate voltage $\left(V_{\mathrm{g}}\right)$ using a semiconductor device analyzer (B1500A, Agilent). Sheet resistance $\left(R_{\mathrm{s}}\right)$ was measured by d.c. four probe method in the van der Pauw electrode configuration. Thermopower ( $S$-value) was measured by giving a temperature difference $(\Delta T)$ of $\sim 4 \mathrm{~K}$ in the film using two Peltier devices while the actual temperatures of both sides of $\mathrm{VO}_{2}$ film surface were monitored by two tiny thermocouples. The thermo-electromotive force $(\Delta V)$ and $\Delta T$ were simultaneously measured, and the $S$-values were obtained from the slope of the $\Delta V-\Delta T$ plots. All the electrical property measurements were performed in an ambient atmosphere. 
Structural analysis: TEM samples of the CAN-gated $\mathrm{VO}_{2}$ TFTs were prepared by a focused-ion-beam (FIB) micro-sampling technique, in which the trilayer structure region of the TFTs was cutout and thinned by FIB (JIB-4600F, JEOL) to obtain samples for cross-sectional observation. The cross-sectional microstructure of $\mathrm{VO}_{2}$ TFTs was examined at RT by HAADF-STEM (JEM-ARM200F, 200 kV, JEOL), where electron incident direction was sapphire [0001]. The ED patterns were observed by nanobeam electron diffraction with spot size of about $1.5 \mathrm{~nm}$ (JEM-2800, $200 \mathrm{kV}$, JEOL).

\section{Acknowledgements}

This work was supported by Grant-in-Aid for Scientific Research on Innovative Areas (25106007) from JSPS and Nanotechnology Platform Program (12024046) of MEXT. HO was supported by JSPS KAKENHI for Scientific Research A (25246023). TT was supported by JSPS KAKENHI for Young Scientists B (24760533) and the Network Joint Research Center for Materials and Devices. 
[1] T. Norby, M. Widerøe, R. Glöckner, Y. Larring, Dalton Trans. 2004, 19, 3012.

[2] A. Janotti, C. G. Van de Walle, Nature Mater. 2007, 6, 44.

[3] C. G. Granqvist, Handbook of Inorganic Electrochromic Materials, Elsevier, Amsterdam, 1995.

[4] C. Kilic, A. Zunger, Appl. Phys. Lett. 2002, 81, 73.

[5] C. H. Ahn, J.-M. Triscone, J. Mannhart, Nature 2003, 424, 1015.

[6] K. Ueno, S. Nakamura, H. Shomotani, A. Ohtomo, N. Kimura, T. Nojima, H. Aoki, Y. Iwasa, M. Kawasaki, Nat. Mater. 2008, 7, 855.

[7] K. Vanheusden, W. L. Warren, R. A. B. Devine, D. M. Fleetwood, J. R. Schwank, M. R. Shaneyfelt, P. S. Winokur, Z. J. Lemnios, Nature 1997, 386, 587.

[8] J. Yoon, W. Hong, M. Jo, G. Jo, M. Choe, W. Park, J. I. Sohn, S. Nedic, H. Hwang, M. E. Welland, T. Lee, ACS Nano 2011, 5, 558.

[9] J. B. Goodenough, R. Manoharan, M. Paranthaman, J. Am. Chem. Soc. 1990, 112, 2076.

[10] P. G. Dickens, S. J. Hibble, R. H. Jarman, J. Electron. Mater. 1981, $10,999$.

[11] F. J. Morin, Phys. Rev. Lett. 1959, 3, 34.

[12] J. B. Goodenough, J. Solid State Chem. 1971, 3, 490.

[13] J. Wei, H. Ji, W. Guo, A. H. Nevidomskyy, D. Natelson, Nature Nanotechnol. 2012, 7, 357.

[14] M. Nakano, K. Shibuya, D. Okuyama, T. Harano, S. Ono, M. Kawasaki, Y. Iwasa, Y. Tokura, Nature 2012, 487, 459.

[15] J. Jeong, N. Aetukuri, T. Graf, T. D. Schladt, M. G. Samant, S. S. P. Parkin, Science 2013, $339,1402$.

[16] T. Nan, M. Liu, W. Ren, Z-G. Ye, N. X. Sun, Sci. Rep. 2014, 4, 5931.

[17] A. M. Chippindale, P. G. Dickens, A. V. Powell, J. Solid State Chem. 1991, 93, 526.

[18] V. Andreev, V. Kapralova, V. Klimov, Phys. Solid State 2007, 49, 2318.

[19] C. Wu, F. Feng, J. Feng, J. Dai, L. Peng, J. Zhao, J. Yang, C. Si, Z. Wu, Y. Xie, J. Am. Chem. Soc. 2011, 133, 13798.

[20] H. Ohta, Y. Sato, T. Kato, S. W. Kim, K. Nomura, Y. Ikuhara, H. Hosono, Nature Commun. 2010, 1, 118; H. Ohta, T. Mizuno, S. Zheng, T. Kato, Y. Ikuhara, K. Abe, H. Kumomi, K. Nomura, H. Hosono, Adv. Mater. 2012, 24, 740.

[21] C. Ko, Z. Yang, S. Ramanathan, ACS Appl. Mater. Interfaces 2011, 3, 3396.

[22] T. Katase, K. Endo, H. Ohta, Phys. Rev. B 2014, 90, 161105(R).

[23] H. Ohta, S.-W. Kim, Y. Mune, T. Mizoguchi, K. Nomura, S. Ohta, T. Nomura, Y. Nakanishi, Y. Ikuhara, M. Hirano, H. Hosono, K. Koumoto, Nature Mater. 2007, 6, 129. 
[24] Y. Filinchuk, N. A. Tumanov, V. Ban, H. Ji, J. Wei, M. W. Swift, A. H. Nevidomskyy, D. Natelson, J.Am. Chem. Soc. 2014, 136, 8100.

[25] J. Karel, C. E. ViolBarbosa, J. Kiss, J. Jeong, N. Aetukuri, M. G. Samant, X. Kozina, E. Ikenaga, G. H. Fecher, C. Felser, S. S. P. Parkin, ACS Nano 2014, 8, 5784.

[26] R. D. Shannon, Acta Cryst. 1976, A32, 751.

[27] H. Ohta, Y. Masuoka, R. Asahi, T. Kato, Y. Ikuhara, K. Nomura, H. Hosono, Appl. Phys. Lett. 2009, 95, 113505.

[28] J. H. Park, J. M. Coy, T. S. Kasirga, C. Huang, Z. Fei, S. Hunter, D. H. Cobden, Nature 2013, 500, 431.

[29] H. Amano, M. Kito, K. Hiramatsu, I. Akasaki, Jpn. J. Appl. Phys. 1989, 28, L2114.

[30] S. Nakamura, N. Iwasa, M. Senoh, T. Mukai, Jpn. J. Appl. Phys. 1992, 131, 1258. 


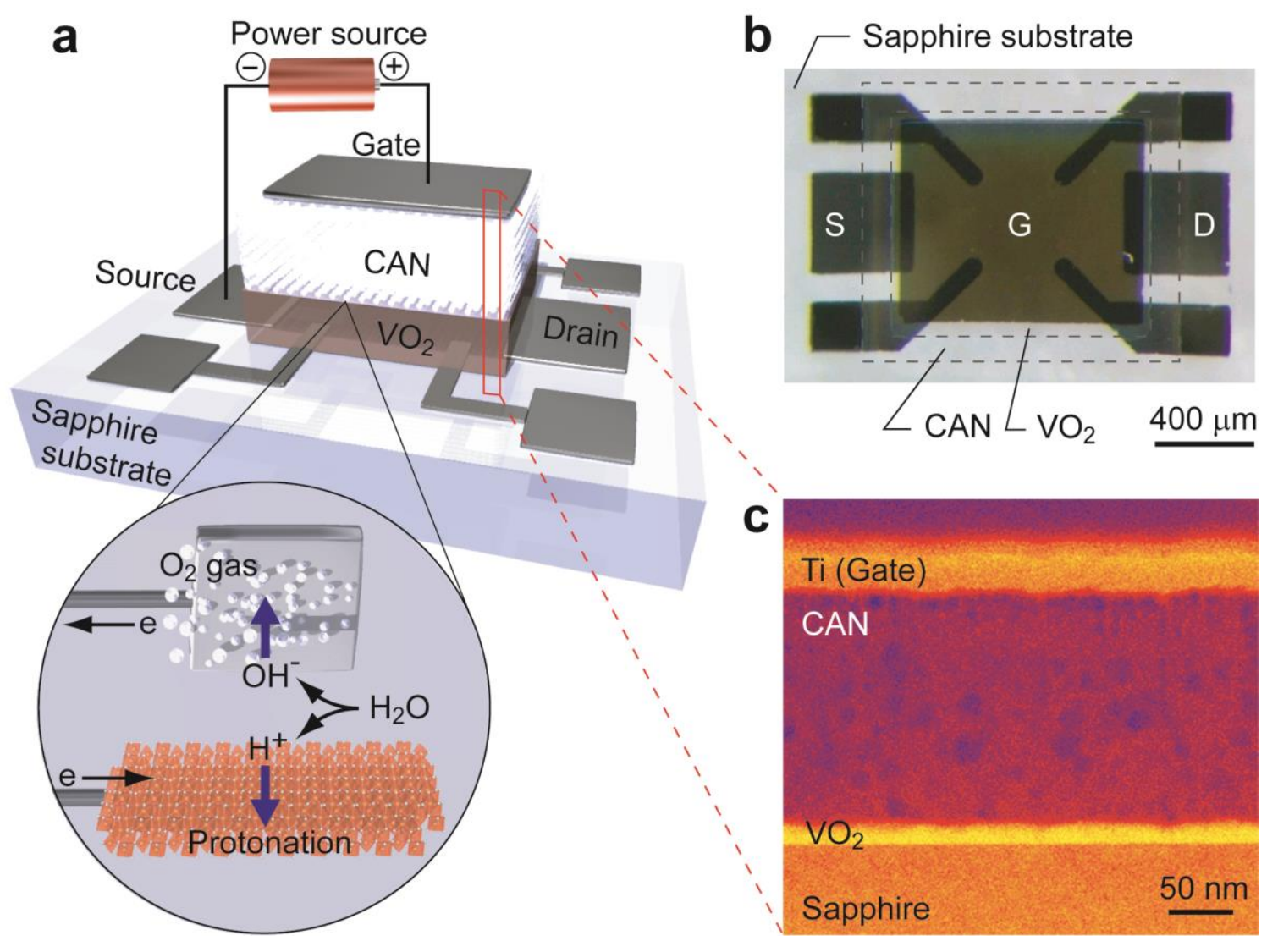

Figure 1. Water-electrolysis-induced protonation of $\mathrm{VO}_{2}$ thin film. (a) Schematic of the thin film transistor (TFT) structure composed of Ti electrodes / water-infiltrated CAN gate insulator / $\mathrm{VO}_{2}$ epitaxial film on sapphire substrate. Magnified image illustrates the principle of electrolysis-induced protonation of the $\mathrm{VO}_{2}$ film. During positive gate voltage $\left(V_{\mathrm{g}}\right)$ application, $\mathrm{H}^{+}$and $\mathrm{OH}^{-}$ions, which are produced by water electrolysis in the CAN film (nanosized electrochemical cell), move to the surfaces of $\mathrm{VO}_{2}$ film and $\mathrm{Ti}$ gate electrode, respectively. $\mathrm{H}^{+}$ions penetrate into the $\mathrm{VO}_{2}$ crystal $\left(\mathrm{H}_{x} \mathrm{VO}_{2}\right)$ and $\mathrm{OH}^{-}$ions become $\mathrm{O}_{2}$ gas at each interface. (b) Optical micrograph of the $\mathrm{VO}_{2}$-based TFT. Channel width and length are $400 \mu \mathrm{m}$ and $800 \mu \mathrm{m}$, respectively. (c) Cross-sectional HAADF-STEM image of Ti (50 nm) / CAN (200 nm) / $\mathrm{VO}_{2}(20 \mathrm{~nm}) /$ sapphire substrate. Nanopores with 10-20 nm diameters appear as dark contrasts in the CAN film. 

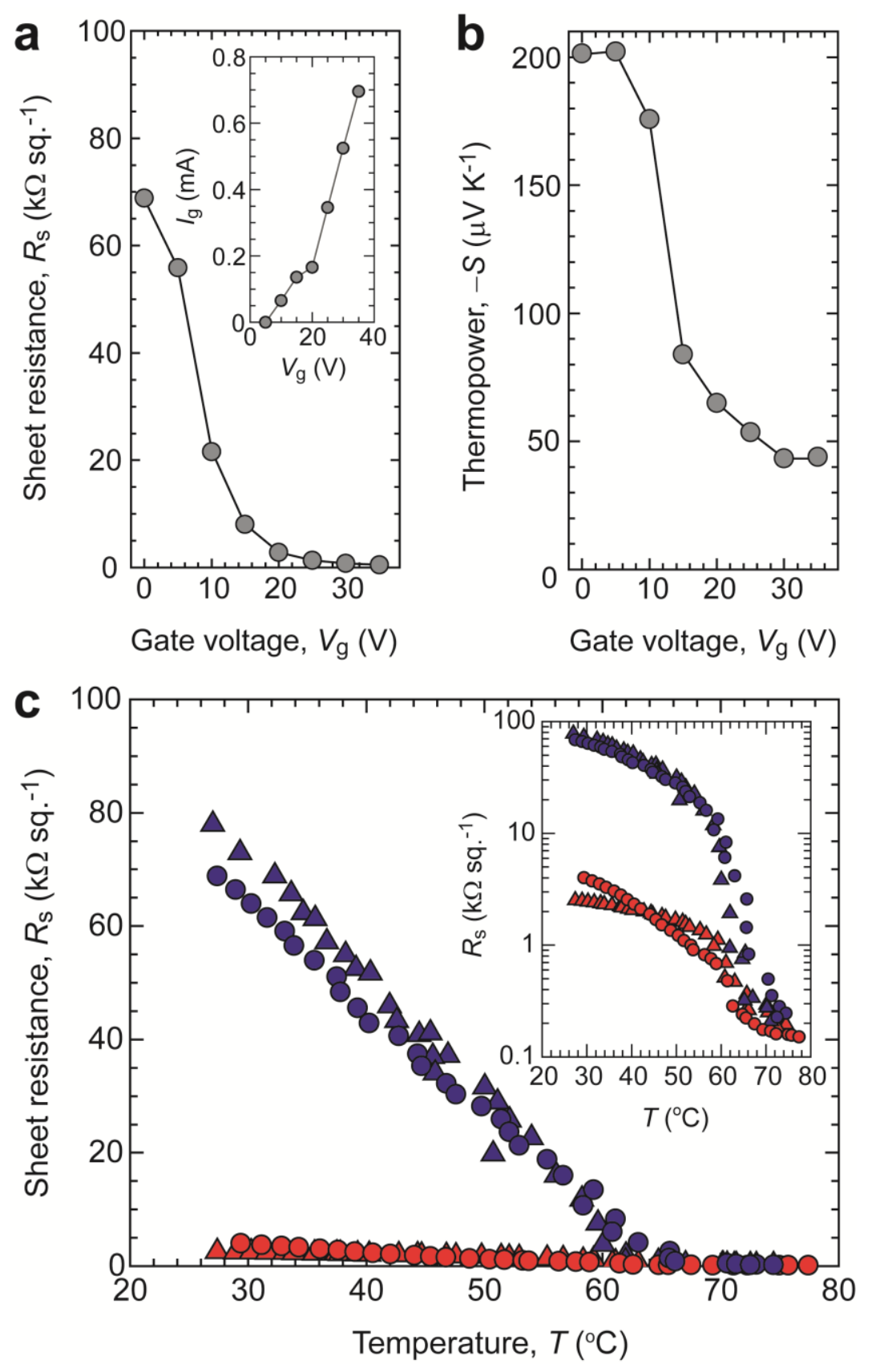

Figure 2. Protonation-driven metal-insulator conversion of $\mathrm{VO}_{2}$ thin film. (a) Sheet resistance $\left(R_{\mathrm{S}}\right)$ as a function of $V_{\mathrm{g}}$ up to $+35 \mathrm{~V}$, where $R_{\mathrm{s}}$ was measured after holding the $V_{\mathrm{g}}$ application for 10 minutes. Inset shows $V_{\mathrm{g}}$ dependence of mean gate current (三ion current, $I_{\mathrm{g}}$ ) during the $V_{\mathrm{g}}$ application. $I_{\mathrm{g}}$ exponentially increased with $V_{\mathrm{g}} \geq+10 \mathrm{~V}$ due to water-electrolysis-induced protonation of $\mathrm{VO}_{2} . R_{\mathrm{s}}$ simultaneously decreased with $V_{\mathrm{g}} \geq+10 \mathrm{~V}$, indicating that the electronic state of $\mathrm{VO}_{2}$ was altered from an insulator to a metal by water-electrolysis-induced 
protonation. (b) Thermopower $(S)$ as a function of $V_{\mathrm{g}}$ at RT. $|S|$ decreased with $V_{\mathrm{g}} \geq+10 \mathrm{~V}$ and was saturated at $43 \mu \mathrm{V} \mathrm{K}{ }^{-1}$, which is close to that of metallic $\mathrm{H}_{x} \mathrm{VO}_{2}\left(\sim 35 \mu \mathrm{V} \mathrm{K}^{-1}\right){ }^{[19]}$ (c) Temperature dependence of $R_{\mathrm{s}}$ measured before (blue closed circles) and after applying $V_{\mathrm{g}}$ alternately at $+20 \mathrm{~V}$ (red closed symbols) and $-20 \mathrm{~V}$ (blue closed triangles) at room temperature (RT). Each curve was measured after switching off the $V_{\mathrm{g}}$. Inset shows logarithmic $R_{\mathrm{S}}$ vs. $T$. On/off ratio was $\sim 30$ at RT. 

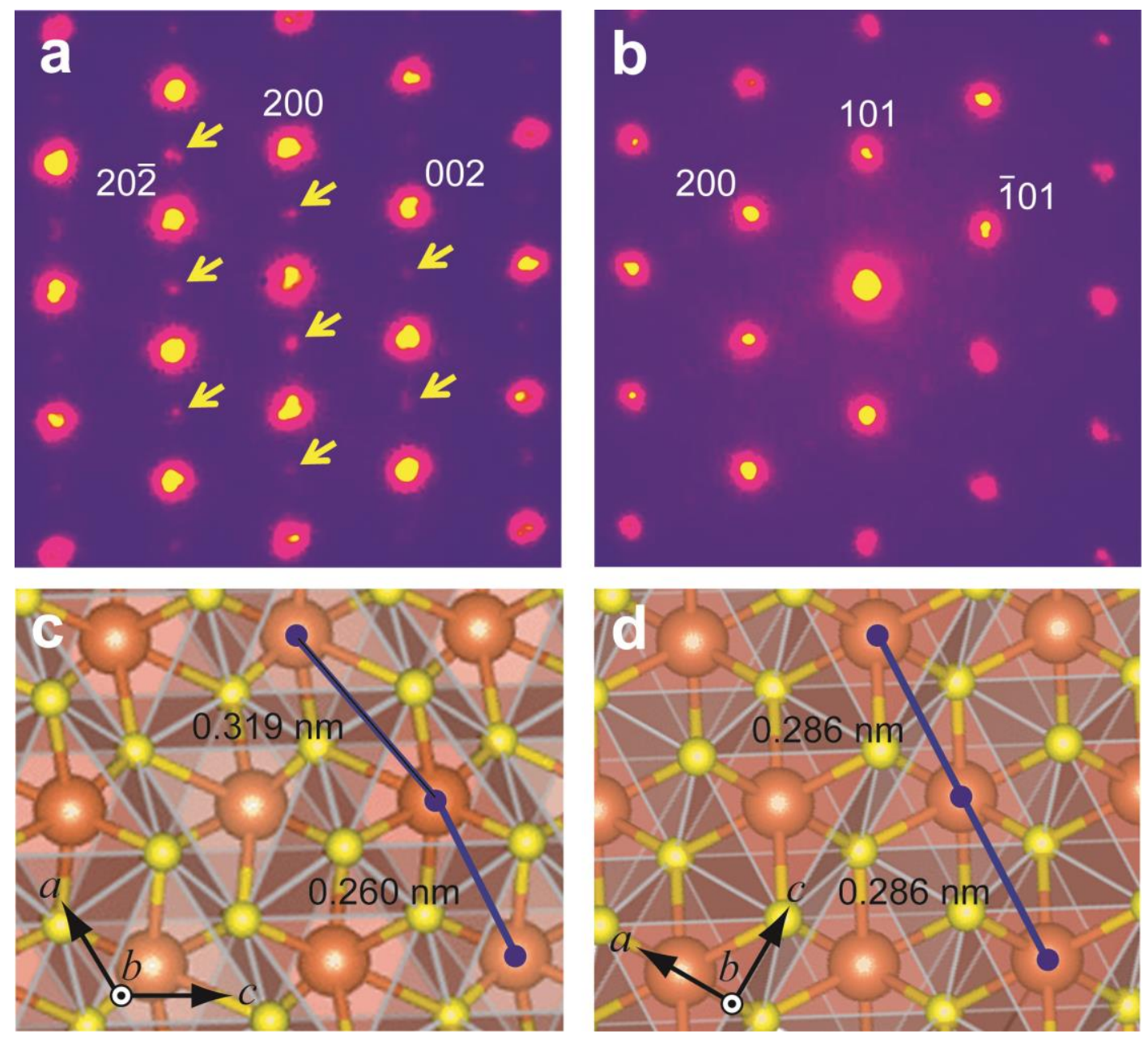

Figure 3 Structural analysis of protonated $\mathrm{VO}_{2}$ thin film. Nano-beam electron diffraction patterns of (a) as-prepared and (b) protonated $\mathrm{VO}_{2}$ films. The main diffraction indices are noted above the corresponding diffraction spots. Arrows in (a) indicate the diffraction spots due to vanadium-ion dimerization of monoclinic $\mathrm{VO}_{2}$. Spots from the superstructure disappeared upon protonation (b), indicating the structural change from monoclinic to tetragonal phase. Schematic of the crystal structures for (c) monoclinic and (d) tetragonal phases. Brown and yellow denote vanadium and oxygen ions, respectively. 

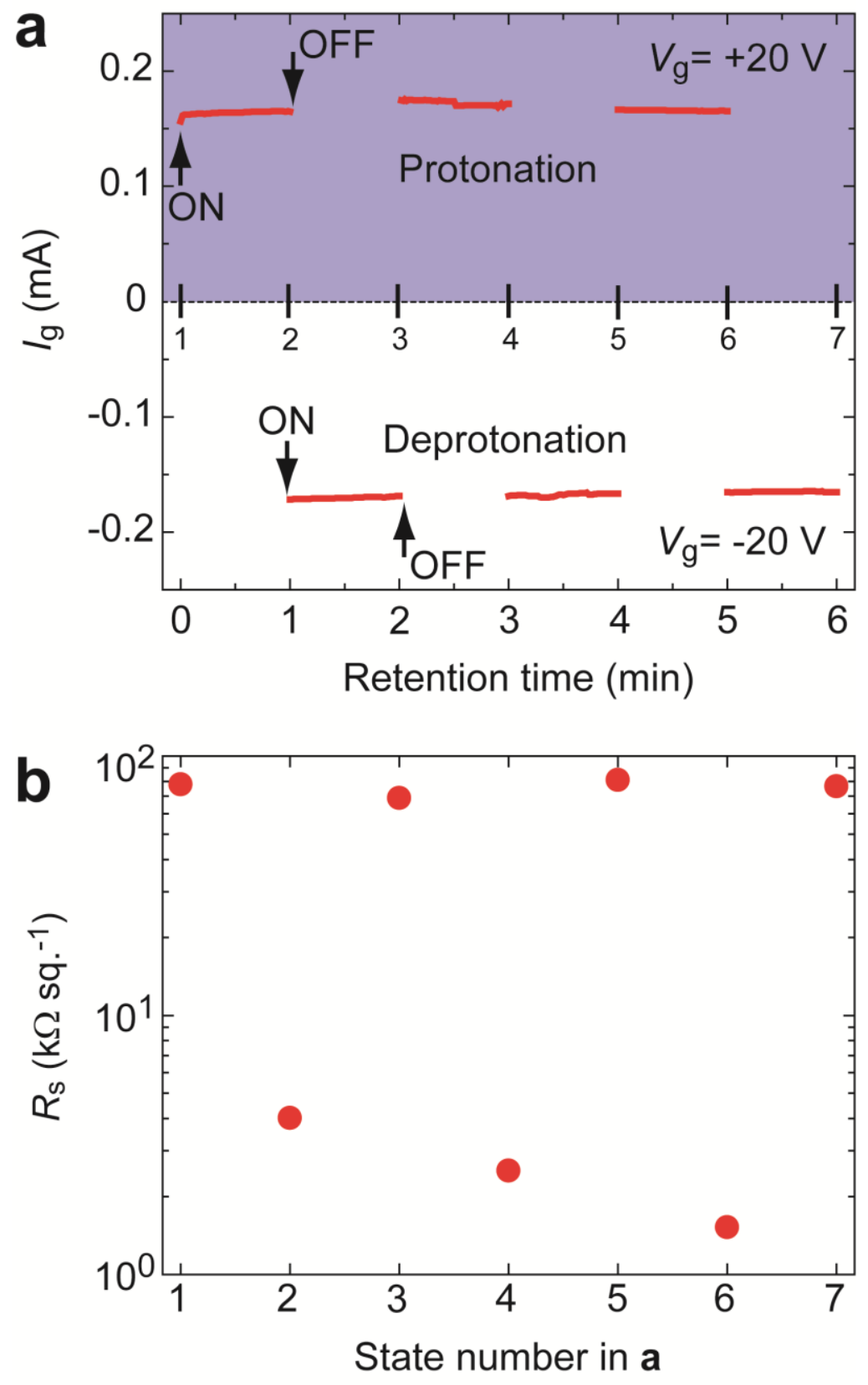

Figure 4 Reversible metal-insulator conversion of $\mathrm{VO}_{2}$ thin film at room temperature. (a) Retention time dependence of $I_{\mathrm{g}}$ during the $V_{\mathrm{g}}$ application. $\mathrm{VO}_{2}$ thin film was protonated at $V_{\mathrm{g}}=+20 \mathrm{~V}$, whereas deprotonation occurs at $V_{\mathrm{g}}=-20 \mathrm{~V}$. (b) $R_{\mathrm{s}}$ modulation at room temperature, which was measured for each state in (a). 